\title{
A novel variant of the CASQ2 gene in a Chinese family with catecholaminergic polymorphic ventricular tachycardia
}

\author{
Lu Gao ${ }^{1 *}$, Lang Cui ${ }^{1 *}$, Lihui Zheng ${ }^{2}$, Zhihui Zhao ${ }^{1}$, Qirui $\mathrm{Li}^{1}$, \\ Xia Yu${ }^{1}$, Jiahui Wang ${ }^{3}$, Yue Yuan ${ }^{1}$ \\ ${ }^{1}$ Beijing Children's Hospital, Capital Medical University, National Center for Children's Health, China \\ ${ }^{2}$ Fuwai Hospital, Chinese Academy of Medical Sciences, Beijing, China \\ ${ }^{3}$ Sinopath Diagnostics, Beijing, China
}

Catecholaminergic polymorphic ventricular tachycardia (CPVT) is an inherited cardiac disorder which is potentially lethal and is characterized by episodic bidirectional or polymorphic ventricular tachycardia during adrenergic stimulation, without apparent structural heart disease. The prevalence of this rare fatal disease has been estimated to be approximately 1:10000 [1]. However, early diagnosis of CPVT is difficult due to a patients' normal heart structure and generally normal resting electrocardiogram. Gene testing is particularly important in diagnosis, and early diagnosis and treatment can effectively prevent sudden death in children [2].

Regulation of calcium flux in the heart is a key process that affects cardiac excitability and contractility. Mutations in gene encoding ryanodine receptor 2 (RYR2) and calsequestrin 2 (CASQ2) are the most common causes of cardiomyocyte calcium imbalance [3]. This study documents the case of a Chinese family with CPVT. Clinical and exercise stress testing results of the patients were analyzed in detail, and targeted exome sequencing technology was applied.

The diagnosis of CPVT can be based on: 1) In the presence of a structurally normal heart and coronary arteries, normal electrocardiogram, unexplained exercise or catecholamine-induced bidirectional ventricular tachycardia (BVT), polymorphic premature ventricular contractions (PVCs) or ventricular tachycardia in individuals $<40$ years old; 2) A pathogenic mutation; 3) Family members of a CPVT index case with a normal heart who manifests exercise-induced PVCs or BVT/PVT [4]. This Chinese family has 5 children, 3 daughters and 2 sons. The proband is the second daughter and her two younger brothers, who all met the diagnostic criteria for CPVT.

Many previous studies have confirmed syncope as the main clinical symptom of CPVT $[5,6]$. The 12-year-old proband experienced recurrent syncope 5-6 times per year since the age of 5 , and was diagnozed with epilepsy. She failed to respond to oral antiepileptic medication for seizures. When she was 12 years old, ventricular tachycardia was demonstrated in a routine $24 \mathrm{~h}$ Holter examination. During exercise stress testing, polymorphic ventricular arrhythmia was induced at a heart rate of $126 \mathrm{bpm}$ in stage III. One of the proband brothers, the fourth child in this family, was 10 years old and experienced syncope during exercise at 8 years old. During exercise stress testing, polymorphic ventricular arrhythmia was induced at a heart rate of $125 \mathrm{bpm}$ in stage IV. The other younger brother of the proband is 5 years old, and had experienced syncope 3 days prior to exercise. Exercise stress testing was also performed; when the heart rate reached $125 \mathrm{bpm}$ in stage IV, ventricular premature beats began to appear. No abnormality in the proband or her 2 younger brothers was detected on physical examination, echocardiography, electrocardiography or regarding cerebral blood flow. In each case, the impact of drugs and electrolyte disorder could be excluded. Exercise stress tests of the proband's parents and sisters were negative.

Address for correspondence: Dr. Yue Yuan, Beijing Children's Hospital, Capital Medical University, National Center for Children's Health, China, 100045, Postal code: 100045, China, tel: +8613366971016, e-mail: yuanyuebj22@163.com

Received: 8.08.2018 Accepted: 2.09.2018

*Lu Gao and Lang Cui are both the first author. 


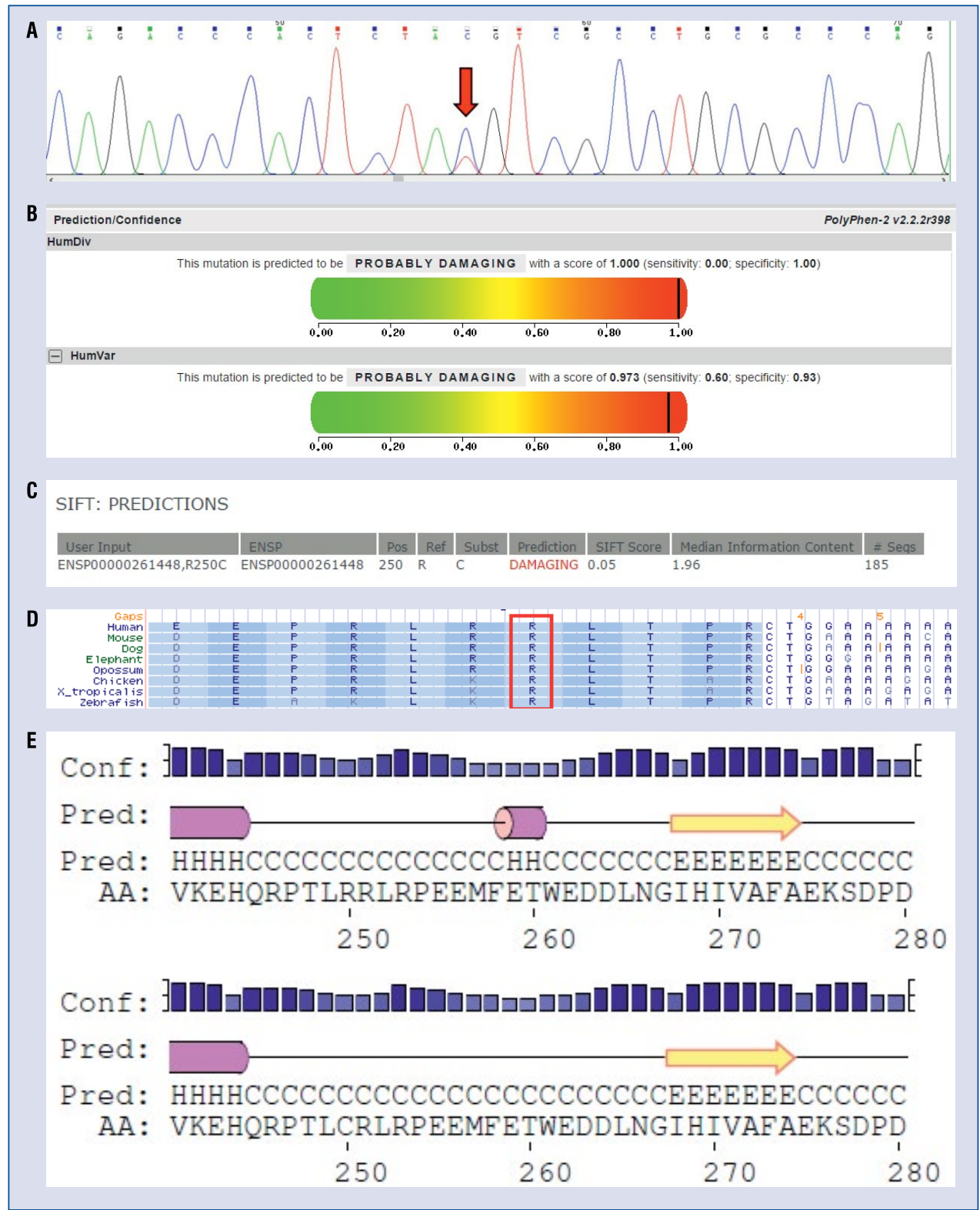

Figure 1. A. Sequencing results and bioinformatic analysis of the gene mutation in this study; B. Polyphen-2 score of the novel damaging mutation $c .748 C>T$ (p.R250C); C. SIFT score of the novel damaging mutation $c .748 C>T$ (p.R250C); D. The mutation site is conserved across multiple species; E. PSIPRED prediction of the polypeptide chain secondary structure.

To further confirm the diagnoses, Next Generation Sequencing technology was applied for the proband. The QIAamp DNA Mini Kit (Qiagen, Germany) was used to isolate genomic DNA from peripheral blood leukocytes. Comprehensive detection of genetic cardiovascular diseases was performed using the SeqCap EZ Choice XL Library (Roche NimbleGen). Next, exome sequencing data were generated by $150 \mathrm{bp}$ end reads on the Illumina Hiseq 4000 and analyzed in house pipeline. Paired- end reads in the FASTQ files were mapped on to the human reference genome sequence (GRCh37/ hg19), revealing two heterozygous mutations c. $97 \mathrm{C}>\mathrm{T}$ (p.R33X) and c.748C $>\mathrm{T}(\mathrm{p} . \mathrm{R} 250 \mathrm{C})$ in the CASQ2 gene. Blood samples were collected from the proband's family for gene sequencing.

Sanger sequencing confirmed that mutation c. $97 \mathrm{C}>\mathrm{T}(\mathrm{p} . \mathrm{R} 33 \mathrm{X})$ in the CASQ2 gene was inherited from the proband's father. This nonsense mutation leads to early termination of protein poly- 
peptide synthesis and is a known mutation reported by the Human Gene Mutation Database [7].

This article will focus primarily on the c.748C $>$ T mutation (Fig. 1A), in which the arginine at codon 250 (p.R250C) was substituted by cysteine. This mutation was found in the proband's mother and had not been previously reported; the proband and her 2 younger brothers were all heterozygous carriers. The variant c. $748 \mathrm{C}>\mathrm{T}$ (p.R250C) was not observed in approximately 10,000 samples in the Sinopath laboratory database (undisclosed) from individuals of Chinese backgrounds and some databases like ExAC/ESP, indicating that it is not a common benign variant or a polymorphism in these populations. Silico algorithms PolyPhen-2, SIFT and Mutation_Taster all predicted it to be deleterious. In addition, it was predicted to cause possible damage by Polyphen-2 with a score of 0.973 (Fig. 1B), and SIFT predicted this mutation to be damaging, with a score of 0.05 (Fig. 1C). According to the University of California Santa Cruz (UCSC) database, this mutation site is conserved across multiple species (Fig. 1D). Use of PSIPRED to predict the secondary structure of the polypeptide chain determined that there was a novel alpha helix at the original 260 amino acid position. However, the mutation in the gene resulted in the disappearance of the alpha helix in the corresponding protein polypeptide chain, most likely further affecting its corresponding protein space structure or related protein function (Fig. 1E).

The structure and function of proteins is determined by some key amino acids. When these amino acids are changed, the proteins also change. If the site of a mutation is in a very conservative position, then the variation may have a greater impact on the structure and function. The site of the p.R250C mutation in the UCSC database query shows that it is conserved across many species, so it is highly likely that the changes may have an impact on protein function.

Pharmacological therapy was prescribed to of the 3 subjects. The proband and her 2 younger brothers were all given oral beta-blocker metopro- lol at $2 \mathrm{mg} / \mathrm{kg}$. After pharmacological therapy, the patients did not faint again.

In this study, a pedigree of catecholaminesensitive pleomorphic ventricular tachycardia based was diagnosed from clinical manifestations, exercise stress testing and genetic analysis. A new heterozygous mutation site c. $748 \mathrm{C}>\mathrm{T}$ in the CASQ2 gene was detected, which resulted in the substitution of arginine at codon 250 by cysteine. These results extend the genetic mutation spectrum of CASQ2 and demonstrate that targeted exome sequencing is an effective method for the diagnosis of CPVT in clinical practices.

Conflict of interest: None declared

\section{References}

1. Leenhardt A, Denjoy I, Guicheney P. Catecholaminergic polymorphic ventricular tachycardia. Circ Arrhythm Electrophysiol. 2012; 5(5): 1044-1052, doi:10.1161/circep.111.962027.

2. Behere SP, Weindling SN. Catecholaminergic polymorphic ventricular tachycardia: An exciting new era. Ann Pediatr Cardiol. 2016; 9(2): 137-146, doi: 10.4103/0974-2069.180645, indexed in Pubmed: 27212848.

3. Venetucci L, Denegri M, Napolitano C, et al. Inherited calcium channelopathies in the pathophysiology of arrhythmias. Nat Rev Cardiol. 2012; 9(10): 561-575, doi: 10.1038/nrcardio.2012.93, indexed in Pubmed: 22733215.

4. Priori SG, Wilde AA, Horie M, et al. Executive summary: HRS/ /EHRA/APHRS expert consensus statement on the diagnosis and management of patients with inherited primary arrhythmia syndromes. Heart Rhythm. 2013; 10(12): e85-108, doi: 10.1016/j. hrthm.2013.07.021, indexed in Pubmed: 23916535.

5. Ge HY, Li XM, Jiang H, et al. [Clinical challenges in the management of catecholaminergic polymorphic ventricular tachycardia in children]. Zhonghua Er Ke Za Zhi. 2017; 55(12): 926-931, indexed in Pubmed: 29262473.

6. Hazle MA, Shellhaas RA, Bradley DJ, et al. Arrhythmogenic channelopathy syndromes presenting as refractory epilepsy. Pediatr Neurol. 2013; 49(2): 134-137, doi: 10.1016/j.pediatrneurol.2013.03.017, indexed in Pubmed: 23859862.

7. Paludan-Müller C, Ahlberg G, Ghouse J, et al. Integration of 60,000 exomes and ACMG guidelines question the role of Catecholaminergic Polymorphic Ventricular Tachycardia-associated variants. Clin Genet. 2017; 91(1): 63-72, doi: 10.1111/cge.12847, indexed in Pubmed: 27538377. 